\title{
ON THE VANISHING AND THE POSITIVITY OF INTERSECTION MULTIPLICITIES OVER LOCAL RINGS WITH SMALL NON COMPLETE INTERSECTION LOCI
}

\author{
KAZUHIKO KURANO
}

\section{Introduction}

Throughout this paper $A$ is a commutative Noetherian ring of dimension $d$ with the maximal ideal $\mathrm{m}$ and we assume that there exists a regular local ring $S$ such that $A$ is a homomorphic image of $S$, i.e., $A=S / I$ for some ideal $I$ of $S$. Furthermore we assume that $A$ is equi-dimensional, i.e., $\operatorname{dim} A=\operatorname{dim} A / \mathfrak{p}$ for any minimal prime ideal $\mathfrak{p}$ of $A$. We put

$$
V=\left\{\mathfrak{p} \in \operatorname{Spec}(A) \mid\left(I / I^{2}\right) \otimes_{A} A_{\mathfrak{p}} \text { is not an } A_{\mathfrak{p}} \text {-free module }\right\} .
$$

Recall that $V$ coincides with the non complete intersection locus of $A$. For convenience, we set $\operatorname{dim} V=-\infty$ when $V$ is empty. Let $M$ and $N$ be finitely generated $A$-modules such that $\ell_{A}\left(M \otimes_{A} N\right)<\infty$, where $\ell_{A}(*)$ stands for the length as an $A$-module.

Throughout this paper we maintain the notation as above unless otherwise specified.

The aim of this paper is to prove the following theorem:

THEOREM 1.1. With notation as above, suppose $\operatorname{pd}_{A}(M)<\infty$ and $\operatorname{pd}_{A}(N)<\infty$, where $\operatorname{pd}_{A}(*)$ is the projective dimension as an A-module. Put

$$
\chi(M, N)=\sum_{i \geq 0}(-1)^{i} \ell_{A}\left(\operatorname{Tor}_{i}^{A}(M, N)\right) .
$$

(1) If $\operatorname{dim} V \leq 1$ and $\operatorname{dim} M+\operatorname{dim} N<d$, then $\chi(M, N)=0$.

(2) Suppose that $A$ is a Gorenstein ring, or a normal domain whose canonical class $\mathrm{cl}\left(K_{A}\right)$ is a torsion element in the divisor class group $\mathrm{Cl}(A)$ of $A$. Furthermore suppose that $d$ is an odd number. Then $\chi(M, N)=0$ if $\operatorname{dim} V \leq 2$ and $\operatorname{dim} M+\operatorname{dim} N<d$.

(3) Assume that $\operatorname{dim} V=0, \operatorname{dim} M+\operatorname{dim} N=d$, $\operatorname{depth} M=\operatorname{dim} M$, and

Received March 15, 1993. 
one of the following two conditions is satisfied:

(a) A contains a field.

(b) $p^{\nu} N=0$ for $\nu \gg 0$, where $p>0$ is the characteristic of the residue class field $A / \mathrm{m}$.

Then we get $\chi(M, N)>0$.

Originally this kind of problem was raised in 1965 by Serre [17], asking

(v) if $\operatorname{dim} M+\operatorname{dim} N<d$, then $\chi(M, N)=0$,

(p) if $\operatorname{dim} M+\operatorname{dim} N=d$, then $\chi(M, N)>0$

for finitely generated $A$-modules $M$ and $N$ satisfying $0<\ell_{A}\left(M \otimes_{A} N\right)<\infty$ especially when $A$ is a regular local ring. Serre proved them in [17] in the case where $A$ is an unramified regular local ring. After that (v) was solved affirmatively for any regular local ring by Gillet-Soulé [7] and Roberts [14] independently.

If $A$ is any local ring, $M$ is a finitely generated $A$-module of finite projective dimension, and $N$ is any finitely generated $A$-module such that $\ell_{A}\left(M \otimes_{A} N\right)<\infty$, then we can define $\chi(M, N)$. It is natural to ask whether the statements (v) and (p) hold in this generality. But, Dutta, Hochster and MacLaughlin [5] succeeded to construct counterexamples to both (v) and (p). In their examples, $A$ is a hypersurface of dimension 3 and the projective dimension of $N$ is infinite.

Therefore, when $A$ is not a regular local ring, it seems to be necessary to assume that both $M$ and $N$ have finite projective dimension. In fact Roberts proved the statement (v) not only for regular local rings, but also when $M$ and $N$ are modules of finite projective dimension over a complete intersection or a local ring whose singular locus has dimension at most one. (1) of Theorem 1.1 is an extension of the results due to Gillet-Soule and Roberts to the case where the non complete intersection locus of a given equi-dimensional local ring has dimension at most one. In the same situation as in (3) of Theorem 1.1, $\chi(M, N)>0$ has been already proved for a regular local ring ([4]) or a certain complete intersection ([10]).

The main tool to prove Theorem 1.1 is a localized Chern character (see Section 18 in [6]). We shall prove (1), (2) and a part of (3) of Theorem 1.1 in the next section. The formal arguments with respect to localized Chern characters enable us to prove (1) and (2) of Theorem 1.1. Using "intersection theory", one can prove (3) of Theorem 1.1 if $A$ does not contain the field of rational numbers. However we need complicated, but formal arguments to reduce the case of characteristic zero to the case of positive characteristic. We shall prove (3) of Theorem 1.1 in 
Section 2 when $A$ does not contain the field of rational numbers $\mathbf{Q}$, and in Section 3 when $A$ contains $\mathbf{Q}$.

Throughout this paper we put $X=\operatorname{Spec}(A), Z=\operatorname{Supp}(M), W=\operatorname{Supp}(N)$. Let $\mathbf{F}$. (resp. G.) be the minimal $A$-free resolution of $M$ (resp. $N)$. $(\operatorname{Supp}(*)$ stands for the support of a given module.)

\section{Proof of (1), (2) and a part of (3) of Theorem 1.1}

This section is devoted to proving (1), (2) and a part of (3) of Theorem 1.1.

First of all, we recall some results from "intersection theory" which we shall use in this section. We refer the reader to Fulton [6] for complete definitions and properties of them.

Let $U$ be a scheme of finite type over a regular scheme.

We denote by $A_{*} U=\bigoplus_{i} A_{i} U$ the Chow group of $U$ and put $A_{*} U_{\mathbf{Q}}=A_{*} U \otimes_{\mathbf{Z}} \mathbf{Q}$ and $A_{i} U_{\mathbf{Q}}=A_{i} U \otimes_{\mathbf{Z}} \mathbf{Q}$, where $\mathbf{Z}$ (resp. $\mathbf{Q}$ ) is the ring of integers (resp. the field of rational numbers). For a closed integral subscheme $C$ of $U$, we denote by [C] the cycle in $A_{*} U_{\mathbf{Q}}$ corresponding to $C$. More generally, for an equidimensional closed subscheme $C$ of $U$, we put

$$
[C]=\sum_{i} \ell_{\mathscr{O}_{C, C_{i}}}\left(\mathfrak{O}_{C, C_{i}}\right) \cdot\left[C_{i}\right]
$$

where the above sum runs over all irreducible components of $C$.

For a proper (resp. flat) morphism $i: C \rightarrow U$, one can define the homomorphism $i_{*}: \mathrm{A}_{*} C \rightarrow \mathrm{A}_{*} U$ (resp. $i^{*}: \mathrm{A}_{*} U \rightarrow \mathrm{A}_{*} C$ ).

For a bounded locally free complex $\mathbf{H}$. over $U$ which is exact except for a closed subset $C$ of $U$, one can define an intersection operator

$$
\begin{aligned}
\operatorname{ch}(\mathbf{H} .): A_{*} U_{\mathbf{Q}} & \rightarrow \mathrm{A}_{*} C_{\mathbf{Q}} \\
\alpha & \mapsto \operatorname{ch}(\mathbf{H} .) \cap \alpha
\end{aligned}
$$

called the localized Chern character. We put

$$
\operatorname{ch}(\mathbf{H} .)=\operatorname{ch}_{0}(\mathbf{H} .)+\operatorname{ch}_{1}(\mathbf{H} .)+\cdots,
$$

where $\operatorname{ch}_{i}(\mathbf{H}.) \cap \alpha \in \mathrm{A}_{t-i} C_{\mathbf{Q}}$ if $\alpha \in \mathrm{A}_{t} U_{\mathbf{Q}}$.

$K_{0} U$ denotes the Grothendieck group of coherent sheaves over $U$ and put $\mathrm{K}_{0} U_{\mathbf{Q}}=\mathrm{K}_{0} U \otimes_{\mathbf{Z}} \mathbf{Q}$. For a coherent sheaf $\mathscr{F}$ over $U$, we denote by $[\mathscr{F}]$ the element in $\mathrm{K}_{0} U_{\mathbf{Q}}$ corresponding to $\mathscr{F}$.

We can construct the natural isomorphism $\tau: \mathrm{K}_{0} U_{\mathbf{Q}} \rightarrow \mathrm{A}_{*} U_{\mathbf{Q}}$ of $\mathbf{Q}$-vector 
spaces and $\tau$ is called the Riemann-Roch map.

In this section we shall use these terminologies freely.

Put $\tau([A])=q_{d}+q_{d-1}+\cdots+q_{0}$ with $q_{i} \in \mathrm{A}_{i} X_{\mathbf{Q}}$ in the rest of this section. The next lemma plays an essential role in proving Theorem 1.1.

Lemma 2.1. Let $\mathbf{H}$. be a bounded $A$-free complex which is exact except for a closed subset $C$ of $X$. If $\mathrm{ch}_{t}\left(\mathbf{H}\right.$.) $\cap q_{l} \neq 0$ for some integers $l$ and $t$ satisfying $0 \leq t$ $\leq l \leq d$, then either $l-t \leq \operatorname{dim}(V \cap C)$ or $\operatorname{dim} C \geq d-t$ holds.

Proof. If $X=V$, then the assertion immediately follows from $0 \neq \operatorname{ch}_{t}(\mathbf{H}$.) $\cap$ $q_{l} \in \mathrm{A}_{l-t} C_{\mathbf{Q}}$. Assume $X \neq V$ in the rest of the proof.

Put $Y=\operatorname{Spec}(S)$. Denoting by $\mathbf{P}$. a finite $S$-free resolution of $A$, we get $\tau([A])=\operatorname{ch}(\mathbf{P}.) \cap[Y]$ by the definition of $\tau: \mathrm{K}_{0} X_{\mathbf{Q}} \rightarrow \mathrm{A}_{*} X_{\mathbf{Q}}$ (see [6]). Let $i$ : $X-V \rightarrow X$ and $i^{\prime}: Y-V \rightarrow Y$ be natural inclusions.

Since the following diagram

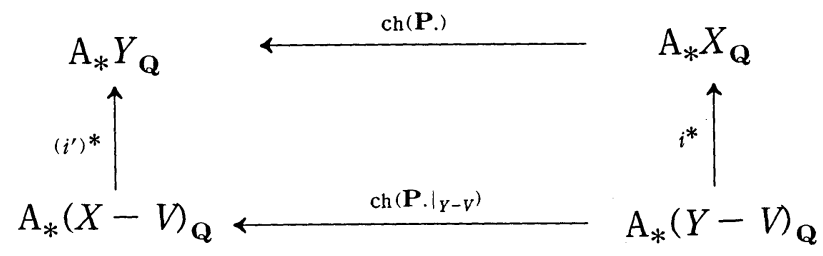

is commutative, we have

$$
\begin{aligned}
& i^{*}\left(q_{d}+q_{d-1}+\cdots+q_{0}\right) \\
= & i^{*}(\operatorname{ch}(\mathbf{P} .) \cap[Y]) \\
= & \operatorname{ch}\left(\mathbf{P} .\left.\right|_{Y-V}\right) \cap[Y-V] .
\end{aligned}
$$

Recall that $V$ coincides with the non complete intersection locus of $X$. Therefore $j: X-V \rightarrow Y-V$ is a locally complete intersection morphism of (constant) codimension $\operatorname{dim} Y-\operatorname{dim} X$ because $X$ is equi-dimensional. Let $\mathscr{E}$ be the normal bundle of $j$. Then $\mathscr{E}$ is a vector bundle over $X$ of (constant) rank $\operatorname{dim} Y-\operatorname{dim} X$. Since $\mathbf{P}$. $\left.\right|_{Y-V}$ is a finite $\mathscr{O}_{Y-V}$-free resolution of $\mathscr{O}_{X-V}$, we have $\operatorname{ch}\left(\left.\mathbf{P} \cdot\right|_{Y-V}\right)=$ $\operatorname{td}(\mathscr{E})^{-1} \cdot j^{!}$(see Corollary 18.1.2 of [6]), where $j^{!}$is the refined Gysin map induced by the locally complete intersection morphism $j$ and $\operatorname{td}(\mathscr{E})$ stands for the Todd class of the vector bundle $\mathscr{E}$ (see [6]). Thus we get

$$
\operatorname{ch}\left(\left.\mathbf{P} \cdot\right|_{Y-V}\right) \cap[Y-V]
$$




$$
\begin{aligned}
& =\operatorname{td}(\mathscr{E})^{-1} \cap j^{!}([Y-V]) \\
& =\operatorname{td}(\mathscr{E})^{-1} \cap[X-V] .
\end{aligned}
$$

le denote by $c_{i}$ the $i$-th Chern class of $\mathscr{E}$. By the definition of $\operatorname{td}(\mathscr{E}),\left(\operatorname{td}(\mathscr{E})^{-1}\right)_{d-l}$ ; a homogeneous polynomial in $c_{1}, c_{2}, \ldots$ of degree $d-l$ with $\operatorname{deg} c_{i}=i$ for each Putting $\left(\operatorname{td}(\mathscr{\mathscr { C }})^{-1}\right)_{d-1}=f_{d-l}\left(c_{1}, c_{2}, \ldots\right)$, we have $i^{*}\left(q_{l}\right)=f_{d-l}\left(c_{1}, c_{2}, \ldots\right) \cap$ $X-V]$.

If $V$ includes $C$, then $\operatorname{dim}(V \cap C) \geq l-t$ follows from $0 \neq \operatorname{ch}_{t}(\mathbf{H}.) \cap q_{l} \in$ ${ }_{l-t} C_{\mathbf{Q}}$.

Next assume $l-t>\operatorname{dim}(V \cap C)$. Then we know that $C-V$ is not empty, ir the following sequence

$$
\mathrm{A}_{l-t}(V \cap C)_{\mathbf{Q}} \rightarrow \mathrm{A}_{l-t} C_{\mathbf{Q}} \stackrel{\left(i^{\prime \prime}\right)^{*}}{\rightarrow} A_{l-t}(C-V)_{\mathbf{Q}} \rightarrow 0
$$

exact and $\mathrm{A}_{l-t} C_{\mathbf{Q}} \neq(0)$. (Note that $\left(i^{\prime \prime}\right)^{*}$ is an isomorphism since $\mathrm{A}_{l-t}(V \cap$ )$_{\mathbf{Q}}=(0)$.) Letting $i^{\prime \prime}: C-V \rightarrow C$ be the natural inclusion, the following diacam

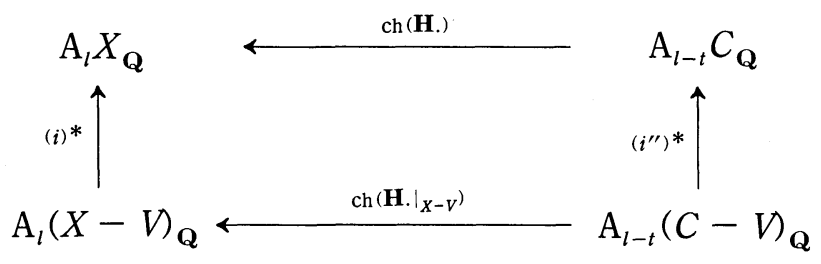

commutative. Since $\left(i^{\prime \prime}\right)^{*}$ is an isomorphism, we obtain

$$
\begin{aligned}
& \left(i^{\prime \prime}\right)^{*}\left(\operatorname{ch}_{t}(\mathbf{H} .) \cap q_{l}\right) \\
= & \operatorname{ch}_{t}\left(\mathbf{H} .\left.\right|_{X-V}\right) \cap i^{*}\left(q_{l}\right) \\
= & \operatorname{ch}_{t}\left(\mathbf{H} .\left.\right|_{X-V}\right) \cap f_{d-l}\left(c_{1}, c_{2}, \ldots\right) \cap[X-V] \\
= & f_{d-l}\left(c_{1}^{\prime}, c_{2}^{\prime}, \ldots\right) \cap \operatorname{ch}_{t}\left(\mathbf{H} .\left.\right|_{X-V}\right) \cap[X-V] \\
\neq & 0,
\end{aligned}
$$

y Proposition 17.3.2 in [6], where we denote by $c_{i}^{\prime}$ the $i$-th Chern class of the ector bundle $g^{*}(\mathscr{E})$ on $C-V$. (Here, $g: C-V \rightarrow X-V$ is the closed immeron.) In particular, we have $\operatorname{ch}_{t}\left(\mathbf{H} .\left.\right|_{X-V}\right) \cap[X-V] \neq 0$. Then the assertion nmediately follows from

$$
\geq \operatorname{codim}(C-V, X-V)=\operatorname{codim}(\overline{C-V}, X) \geq \operatorname{codim}(C, X)=\operatorname{dim} X-\operatorname{dim} C,
$$
here $\overline{C-V}$ is the closure of $C-V$ in the Zariski topology.

Q.E.D. 
Now we start to prove Theorem 1.1.

Proof of (1) of Theorem 1.1. If either $\operatorname{dim} Z$ or $\operatorname{dim} W$ is equal to 0 , then the assertion is obvious. (In fact, if $\operatorname{dim} Z=0$, then we have $\chi(M, N)=\ell_{A}(M)$. $\operatorname{rank}_{A}(N)=0$.) Therefore we may assume $\operatorname{dim} Z>0$ and $\operatorname{dim} W>0$. Here note that, since $d>\operatorname{dim} Z+\operatorname{dim} W>0$, we have $\mathrm{A}_{0} X_{\mathbf{Q}}=(0)$ and, in particular, $q_{0}=0$. Then

$$
\begin{aligned}
\chi(M, N) \cdot[\operatorname{Spec}(A / \mathrm{m})] & =\operatorname{ch}\left(\mathbf{F} . \otimes_{A} \mathbf{G} .\right) \cap \tau([A]) \\
& =\operatorname{ch}\left(\mathbf{F} . \otimes_{A} \mathbf{G} .\right) \cap\left(q_{d}+q_{d-1}+\cdots+q_{1}\right) \\
& =\sum_{l=1}^{d} \operatorname{ch}_{l}\left(\mathbf{F} . \otimes_{A} \text { G. }\right) \cap q_{l}
\end{aligned}
$$

holds by Example 18.3.12 in [6].

Assume $\chi(M, N) \neq 0$. Then, for some integer $l$ such that $1 \leq l \leq d$, $\operatorname{ch}_{l}\left(\mathbf{F} . \otimes_{A}\right.$ G. $) \cap q_{l} \neq 0$ is satisfied. Since

$$
0 \neq \operatorname{ch}_{l}\left(\mathbf{F} . \otimes_{A} \text { G. }\right) \cap q_{l}=\sum_{s+t=l} \operatorname{ch}_{s}(\mathbf{F} .) \cdot \operatorname{ch}_{t}(\mathbf{G} .) \cap q_{l},
$$

one can find non negative integers $s, t$ such that $s+t=l$ and $\operatorname{ch}_{s}(\mathbf{F}$.). $\operatorname{ch}_{t}(\mathbf{G}$. $) \cap q_{l} \neq 0$. In particular, by the commutativity of localized Chern characters (Roberts [14]), we have $\operatorname{ch}_{s}\left(\mathbf{F}\right.$.) $\cap q_{l} \neq 0$ and $\operatorname{ch}_{t}\left(\mathbf{G}\right.$.) $\cap q_{l} \neq 0$.

Note that $\operatorname{dim} Z \leq d-2$ and $\operatorname{dim} W \leq d-2$ since $\operatorname{dim} Z>0, \operatorname{dim} W>0$ and $\operatorname{dim} Z+\operatorname{dim} W<d$. Then we have $\operatorname{ch}_{0}(\mathbf{F})=.\operatorname{ch}_{1}(\mathbf{F})=.\operatorname{ch}_{0}(\mathbf{G}$. $)=$ $\operatorname{ch}_{1}(\mathbf{G})=$.0 (see [13]). Therefore we know $s \geq 2$ and $t \geq 2$ and, therefore, it follows from Lemma 2.1 that $\operatorname{dim} Z \geq d-s$ and $\operatorname{dim} W \geq d-t$. But it contradicts $d>\operatorname{dim} Z+\operatorname{dim} W$.

Q.E.D.

Next we prove (2) of Theorem 1.1.

Proof of (2) of Theorem 1.1. We may assume $\operatorname{dim} Z>0$ and $\operatorname{dim} W>0$ as in the proof of (1) of Theorem 1.1.

First of all, we claim $q_{i}=0$ for each even number $i$ such that $i \geq 4$. When $A$ is a Cohen-Macaulay ring, it is easy to see

$$
\tau\left(\left[K_{A}\right]\right)=q_{d}-q_{d-1}+\cdots+(-1)^{d-i} q_{i}+\cdots+(-1)^{d} q_{0}
$$

by the definition of the Riemann-Roch map $\tau$ ([6]). Therefore we immediately obtain $q_{i}=0$ for any even number $i$ if $A$ is a Gorenstein ring and $d$ is odd. On the other hand, suppose that $A$ is a normal domain whose canonical class $\operatorname{cl}\left(K_{A}\right)$ is a 
torsion element in the divisor class group $\mathrm{Cl}(A)$. Then $q_{d-1}=0$ follows from Lemma 3.5 of [10]. With the same notation as in the proof of Lemma 2.1,

$$
i^{*}\left(q_{d}+q_{d-1}+\cdots+q_{0}\right)=\operatorname{td}(\mathscr{E})^{-1} \cap[X-V]
$$

holds and, therefore, we have $\left(\operatorname{td}(\mathscr{E})^{-1}\right)_{1} \cap[X-V]=i^{*}\left(q_{d-1}\right)=0$. In general, once $\left(\operatorname{td}(\mathscr{E})^{-1}\right)_{1} \cap[X-V]$ coincides with 0 , it is well known that $\left(\operatorname{td}(\mathscr{E})^{-1}\right)_{n} \cap$ $[X-V]=0$ for any odd number $n$ (e.g. see 1.7 in [8]). Therefore we get $i^{*}\left(q_{i}\right)=0$ for any even number $i$. Then the assertion follows immediately from the exact sequence

$$
\mathrm{A}_{*} V_{\mathbf{Q}} \rightarrow \mathrm{A}_{*} X_{\mathbf{Q}} \stackrel{i^{*}}{\rightarrow} \mathrm{A}_{*}(X-V)_{\mathbf{Q}} \rightarrow 0 .
$$

We now start on the proof of (2) of Theorem 1.1. Assume $\chi(M, N) \neq 0$. Then, as in the proof of (1), there exist integers $s, t \geq 2$ such that $\operatorname{ch}_{s}(\mathbf{F}.) \cap q_{l} \neq$ $0, \operatorname{ch}_{t}(\mathbf{G}$. $) \cap q_{l} \neq 0$ and $0 \leq s+t=l \leq d$. As we have already seen, $q_{l}$ is equal to 0 if $l$ is even such that $l \geq 4$. Therefore, we may suppose that $l$ is at least 5 . According to Lemma 2.1, we have only to consider the following four cases:

1) $l-s \leq \operatorname{dim}(V \cap Z)$ and $l-t \leq \operatorname{dim}(V \cap W)$

2) $l-s \leq \operatorname{dim}(V \cap Z)$ and $\operatorname{dim} W \geq d-t$

3) $\operatorname{dim} Z \geq d-s$ and $l-t \leq \operatorname{dim}(V \cap W)$

4) $\operatorname{dim} Z \geq d-s$ and $\operatorname{dim} W \geq d-t$

Since $s+t=l \geq 5$ and $\operatorname{dim} V \leq 2$, the case 1) never happen.

In the case of 2), we have $t=2$ and, therefore, $\operatorname{dim} Z \geq 2$ and $\operatorname{dim} W \geq d-2$ are satisfied. It contradicts $\operatorname{dim} Z+\operatorname{dim} W<d$.

In the same way as the case 2), we can show that the case 3) never happen.

When the case 4) takes place, we have

$$
d>\operatorname{dim} Z+\operatorname{dim} W \geq 2 d-s-t \geq d,
$$

and it is a contradiction.

We have completed the proof of (2) of Theorem 1.1 .

Q.E.D.

Before proving (3) of Theorem 1.1, we make a remark as follows:

Remark 2.2. As in (3) of Theorem 1.1, let $M^{\prime}$ and $N^{\prime}$ be finitely generated modules over a Noetherian local ring $A^{\prime}$ such that depth $M^{\prime}=\operatorname{dim} M^{\prime}, \operatorname{pd}_{A^{\prime}} M^{\prime}$ $<\infty, 0<\ell_{A^{\prime}}\left(M^{\prime} \otimes_{A^{\prime}} N^{\prime}\right)<\infty$. Then we have

$$
\operatorname{depth} A^{\prime}-\operatorname{depth} M^{\prime}=\operatorname{pd}_{A^{\prime}} M^{\prime} \geq \operatorname{dim} N^{\prime}
$$


by the Auslander-Buchsbaum formula and the intersection theorem (cf. [9] and [15]). Furthermore, if $\operatorname{dim} M^{\prime}+\operatorname{dim} N^{\prime}=\operatorname{dim} A^{\prime}$, then $A^{\prime}$ must be a CohenMacaulay ring and the projective dimension of $M^{\prime}$ is equal to $\operatorname{dim} N^{\prime}$. Hence, once $A$ satisfies the assumption of (3) of Theorem 1.1, $A$ must be a Cohen-Macaulay ring and the projective dimension of $M$ is equal to $\operatorname{dim} N$.

In the rest of this section, we prove (3) of Theorem 1.1 in the case where $A$ does not contain the field of rational numbers $\mathbf{Q}$. The third section is devoted to reducing the case where $A$ contains $\mathbf{Q}$ to the positive characteristic case.

Proof of (3) of Theorem 1.1 (in the case where $A$ does not contain $\mathbf{Q}$ ). Let $p>0$ be the characteristic of the residue class field $A / \mathrm{m}$. By our assumpiton, either $A$ contains a field of characteristic $p$ or $p^{\nu}$ kills $N$ for a sufficiently large $\nu$. Therefore, in both cases, $N$ is killed by $p^{\nu}$ for $\nu \gg 0$.

As in the proof of (1), we may assume $\operatorname{dim} Z>0, \operatorname{dim} W>0$ and, therefore, $d>0$. Then we have

$$
\chi(M, N) \cdot[\operatorname{Spec}(A / \mathrm{m})]=\sum_{l=1}^{d} \operatorname{ch}_{l}\left(\mathbf{F} . \otimes_{A} \mathbf{G} .\right) \cap q_{l} .
$$

At first we claim $\operatorname{ch}_{s}(\mathbf{F}.) \cdot \operatorname{ch}_{t}(\mathbf{G}.) \cap q_{l}=0$ for non negative integers $s, t$ and $l$ such that $0 \leq s+t=l<d$. Assume the contrary, i.e., there exist integers $s, t$ and $l$ such that $\operatorname{ch}_{s}(\mathbf{F}.) \cdot \operatorname{ch}_{t}(\mathbf{G}$. $) \cap q_{l} \neq 0$ and $0 \leq s+t=l<d$. In particular, we have $\operatorname{ch}_{s}(\mathbf{F}.) \cap q_{l} \neq 0$ and $\operatorname{ch}_{t}\left(\mathbf{G}\right.$.) $\cap q_{l} \neq 0$. Since $\operatorname{dim} Z<d$ (resp. $\operatorname{dim} W<d$ ), we have $\operatorname{ch}_{0}(\mathbf{F})=.0\left(\right.$ resp. $\left.\operatorname{ch}_{0}(\mathbf{G})=0.\right)$ and, hence, $s \geq 1$ (resp. $t \geq 1$ ). Then Lemma 2.1 implies $\operatorname{dim} Z \geq d-s$ and $\operatorname{dim} W \geq d-t$. Therefore we have

$$
d=\operatorname{dim} Z+\operatorname{dim} W \geq 2 d-s-t=d+(d-l)>d .
$$

It is a contradiction.

As we have seen as above, it holds that

$$
\begin{aligned}
\chi(M, N) \cdot[\operatorname{Spec}(A / \mathrm{m})] & =\operatorname{ch}_{d}\left(\mathbf{F} . \otimes_{A} \mathbf{G} .\right) \cap[X] \\
& =\sum_{s+t=d} \operatorname{ch}_{s}(\mathbf{F} .) \cdot \operatorname{ch}_{t}(\mathbf{G} .) \cap[X] .
\end{aligned}
$$

If $\operatorname{ch}_{s}$ (F.) $\cdot \operatorname{ch}_{t}$ (G.) $\cap[X] \neq 0$, then we have $s \geq \operatorname{codim}(Z, X)=\operatorname{dim} X-\operatorname{dim} Z$ and $t \geq \operatorname{codim}(W, X)=\operatorname{dim} X-\operatorname{dim} W$ (see [12]) since $X$ is equi-dimensional. In this situation we have

$$
d=s+t \geq 2 d-\operatorname{dim} Z-\operatorname{dim} W=d
$$


and, therefore, $s=\operatorname{dim} X-\operatorname{dim} Z=\operatorname{dim} W$ and $t=\operatorname{dim} X-\operatorname{dim} W=\operatorname{dim} Z$. Hence, putting $i=\operatorname{dim} W$ and $j=\operatorname{dim} Z$, it holds that

$$
\chi(M, N) \cdot[\operatorname{Spec}(A / \mathrm{m})]=\operatorname{ch}_{\imath}(\mathbf{F} .) \cdot \operatorname{ch}_{j}(\mathbf{G} .) \cap[X] .
$$

By Example 18.3.12 in [6],

$$
\operatorname{ch}(\mathbf{G .}) \cap \tau([A])=\tau([N]) \text { in } \mathrm{A}_{*} W_{\mathbf{Q}}
$$

holds. Put

$$
\begin{aligned}
\operatorname{Assh}_{A}(N) & =\left\{\mathfrak{p} \in \operatorname{Supp}(N) \mid \operatorname{dim}_{A} N=\operatorname{dim} A / \mathfrak{p}\right\} \\
& =\left\{\mathfrak{p}_{1}, \ldots, \mathfrak{p}_{k}\right\}
\end{aligned}
$$

and denote by $(\tau([N]))_{e}$ the component of $\tau([N])$ of dimension $e$. (Here note that, since $N$ is killed by $p^{\nu}$ for $\nu \gg 0, A / \mathfrak{p}_{h}$ contains a field of characteristic $p$ for any h.) Then we get

$$
\begin{aligned}
(\tau([N]))_{\imath} & =\sum_{h=1}^{k} \ell_{A_{\mathfrak{p} h}}\left(N_{p_{h}}\right) \cdot\left[\operatorname{Spec}\left(A / \mathfrak{p}_{h}\right)\right] \\
& =(\operatorname{ch}(\mathbf{G} .) \cap \tau([A]))_{i} \\
& =\sum_{r=0}^{j} \operatorname{ch}_{r}(\text { G. }) \cap q_{i+r}
\end{aligned}
$$

and, therefore,

$$
\operatorname{ch}_{i}(\mathbf{F} .) \cap(\tau([N]))_{\imath}=\sum_{r=0}^{j} \operatorname{ch}_{\imath}(\mathbf{F} .) \cdot \operatorname{ch}_{r}(\mathbf{G} .) \cap q_{i+r} .
$$

Since $\operatorname{ch}_{s}(\mathbf{F}.) \cdot \operatorname{ch}_{t}(\mathbf{G}$. $) \cap q_{s+t}=0$ for $s+t<d$, we have

$$
\begin{aligned}
& \operatorname{ch}_{i}(\mathbf{F} .) \cap(\tau([N]))_{i} \\
= & \operatorname{ch}_{i}(\mathbf{F} .) \cdot \operatorname{ch}_{j}(\mathbf{G} .) \cap[X] \\
= & \chi(M, N) \cdot[\operatorname{Spec}(A / \mathrm{m})] .
\end{aligned}
$$

Hence we obtain

$$
\begin{aligned}
\chi(M, N) \cdot[\operatorname{Spec}(A / \mathrm{m})] & =\operatorname{ch}_{i}(\mathbf{F} .) \cap \sum_{h=1}^{k} \ell_{A_{\mathfrak{p} h}}\left(N_{\mathfrak{p}_{h}}\right) \cdot\left[\operatorname{Spec}\left(A / \mathfrak{p}_{h}\right)\right] \\
& =\sum_{h=1}^{k} \ell_{A_{\mathrm{v} h}}\left(N_{\mathfrak{p}_{h}}\right) \cdot \operatorname{ch}_{i}\left(\mathbf{F} \cdot \otimes_{A} A / \mathfrak{p}_{h}\right) \cap\left[\operatorname{Spec}\left(A / \mathfrak{p}_{h}\right)\right] .
\end{aligned}
$$

On the other hand, it is known that $\operatorname{ch}_{i}\left(\mathbf{F} . \otimes_{A} A / \mathfrak{p}_{h}\right) \cap\left[\operatorname{Spec}\left(A / \mathfrak{p}_{h}\right)\right]>0$ for each $h$ (see, e.g., [15]) since $\operatorname{ch}\left(A / \mathfrak{p}_{h}\right)=p>0, \mathbf{F} . \otimes_{A} A / \mathfrak{p}_{h}$ is a (not exact) $A / \mathfrak{p}_{h}$-free complex of length $j=\operatorname{dim} N=\operatorname{dim} A / \mathfrak{p}_{h}$, and that is exact except for 
$\mathfrak{m} / \mathfrak{p}_{h}$ (see Remark 2.2). Hence we have got $\chi(M, N)>0$ Q.E.D.

\section{Reduction to the case of positive characteristic}

This section is devoted to proving (3) of Theorem 1.1 in the case where $A$ contains $\mathbf{Q}$. We would like to reduce this case to the case of positive characteristic, in which we have already proved (3) of Theorem 1.1 in this previous section. In such a reduction a famous Hochster's theorem (Theorem 5.2 in [9]) stated below (Theorem 3.2) sometimes plays an essential role. But, in order to prove (3) of Theorem 1.1, we have to make such a reduction preserving $\operatorname{dim} V=0$. In this point of view, Hochster's theorem is not fit to use when we prove (3) of Theorem 1.1. Therefore, first of all, we shall prove Hochster's theorem in a slightly different form (see Proposition 3.3). Using Proposition 3.3, (3) of Theorem 1.1 will be proved in this section.

The author thinks that such a technique of the reduction in this section is (probably) known but, he can not give adequate references. Therefore we shall give precise proofs.

Definition 3.1. Let $\mathfrak{a}$ be an ideal of the polynomial $\operatorname{ring} \mathbf{Z}\left[X_{1}, \ldots, X_{n}\right]$ with $n$ indeterminates $X_{1}, \ldots, X_{n}$. Let $R$ be a commutative ring and $x_{1}, \ldots, x_{n}$ be elements in $R$. We say that $x_{1}, \ldots, x_{n}$ is a solution of $a$ if $f\left(x_{1}, \ldots, x_{n}\right)=0$ is satisfied in $R$ for any $f \in a$.

We sometimes denote $\mathbf{Z}\left[X_{1}, \ldots, X_{n}\right]$ (resp. $x_{1}, \ldots, x_{n}$ ) simply by $\mathbf{Z}[\underline{X}]$ (resp. $\underline{x})$ if no confusion is possible.

Next theorem due to Hochster ([9]) is sometimes called the metatheorem.

THEOREM 3.2 (Hochster). Let a be an ideal of the polynomial ring

$$
\mathbf{Z}\left[X_{1}, \ldots, X_{d}, W_{1}, \ldots, W_{s}\right]
$$

over $\mathbf{Z}$ with indeterminates $X_{1}, \ldots, X_{d}, W_{1}, \ldots, W_{s}$. Suppose that a Noetherian local ring $B$ containing $\mathbf{Q}$ has a solution $\underline{x}, \underline{w}$ of a such that $x_{1}, \ldots, x_{d}$ forms a system of parameters of $B$. Then there exists a Noetherian local ring $C$ which contains a field of positive characteristic and has a solution $\underline{x}^{\prime}, \underline{w^{\prime}}$ of a such that $x_{1}^{\prime}, \ldots, x_{d}^{\prime}$ forms a system of parameters of $C$.

As we noted before, we shall prove this theorem in a slightly different form as follows: 
Proposition 3.3. Let $\mathfrak{a}$ be an ideal of the polynomial ring

$$
\mathbf{Z}\left[Y_{1}, \ldots, Y_{n}, X_{1}, \ldots, X_{d}, G_{1}, \ldots, G_{l}, W_{1}, \ldots, W_{k}\right]
$$

over $\mathbf{Z}$. Suppose that a regular local ring $B$ containing $\mathbf{Q}$ has a solution $\underline{y}, \underline{x}, \underline{g}, \underline{w}$ of a such that $y_{1}, \ldots, y_{n}$ forms a regular system of parameters of $B$ and $\overline{x_{1}}, \ldots, \overline{x_{d}}$ forms a system of parameters of $B /\left(g_{1}, \ldots, g_{l}\right)$, where $\bar{x}_{i}$ stands for the homomorphic image of $x_{i}$ for each $i$. Then there exists a regular local ring $C$ which is essentially of finite type over a field of positive characteristic and has a solution $\underline{y^{\prime}}, \underline{x^{\prime}}, \underline{g^{\prime}}, \underline{w^{\prime}}$ of a such that $y_{1}^{\prime}, \ldots, y_{n}^{\prime}$ forms a regular system of parameters of $C$ and $\overline{x_{1}^{\prime}}, \ldots, \overline{x_{d}^{\prime}}$ forms a system of parameters of $C /\left(g_{1}^{\prime}, \ldots, g_{l}^{\prime}\right)$.

Our first aim of this section is to prove the above proposition.

Since any complete local ring is a homomorphic image of a regular local ring, Theorem 3.2 is an immediate consequence of Proposition 3.3. Furthermore, as we shall see in Remark 3.9, Proposition 3.3 allows us to reduce the case of characteristic zero to the positive characteristic case while preserving homological properties of a given local ring.

Before proving Proposition 3.3, we show several easy Lemmas.

Lemma 3.4. Let $R$ be a regular local ring of dimension $n$ and $\left(g_{1}, \ldots, g_{l}\right)$ be a proper ideal of $R$. Then, for a sufficiently large $k$, there exists an ideal $\mathfrak{b}$ of the polynomial ring

$$
\mathbf{Z}\left[Y_{1}, \ldots, Y_{n}, G_{1}, \ldots, G_{l}, W_{1}, \ldots, W_{k}\right]
$$

which satisfies the following two conditions:

1. There exist $y_{1}, \ldots, y_{n}, w_{1}, \ldots, w_{k} \in R$ such that $y_{1}, \ldots, y_{n}$ forms a regular system of parameters of $R$ and $\underline{y}, \underline{g}, \underline{w}$ is a solution of $\mathfrak{b}$.

2. If a regular local ring $E$ has a solution $\underline{y^{\prime}}, \underline{g^{\prime}}, \underline{w^{\prime}}$ of $\mathfrak{b}$ such that $y_{1}^{\prime}, \ldots, y_{n}^{\prime}$ forms a regular system of parameters of $E$, then $\mathrm{ht}_{E}\left(g_{1}^{\prime}, \ldots, g_{l}^{\prime}\right) \geq \mathrm{ht}_{R}\left(g_{1}, \ldots\right.$, $\left.g_{l}\right)$.

(As we shall see in Lemma 3.7 , one can find an ideal $\mathfrak{b}$ whose solution satisfies $\mathrm{ht}_{E}\left(g_{1}^{\prime}, \ldots, g_{l}^{\prime}\right)=\mathrm{ht}_{R}\left(g_{1}, \ldots, g_{l}\right)$.)

Proof. Put $h=n-\mathrm{ht}_{R}\left(g_{1}, \ldots, g_{l}\right)$. Then one can choose $x_{1}, \ldots, x_{h} \in R$ such that $\operatorname{dim} R /\left(g_{1}, \ldots, g_{l}, x_{1}, \ldots, x_{h}\right)=0$. Replacing $\left(g_{1}, \ldots, g_{l}\right)$ by $\left(g_{1}, \ldots\right.$, $\left.g_{l}, x_{1}, \ldots, x_{h}\right)$, we may suppose that $\mathrm{ht}_{R}\left(g_{1}, \ldots, g_{l}\right)=n$. Then the assertion is obvious because $\sqrt{\left(g_{1}, \ldots, g_{l}\right)}=\left(y_{1}, \ldots, y_{n}\right)$.

Q.E.D. 
Lemma 3.5. Let $R$ be a regular local ring of dimension $n$ and suppose that the following sequence

$$
R^{p} \stackrel{\left(a_{i j}\right)}{\longrightarrow} R^{q} \stackrel{\left(b_{k l}\right)}{\longrightarrow} R^{r}
$$

is exact, where $\left(a_{i j}\right)$ (resp. $\left(b_{k l}\right)$ ) stands for the $R$-linear map corresponding to the $q$ by $p$ matrix $\left(a_{i j}\right)$ (resp. $r$ by $q$ matrix $\left(b_{k l}\right)$ ). Then, for a sufficiently large $s$, there exists an ideal $\mathfrak{b}$ of the polynomial ring over $\mathbf{Z}$ with indeterminates

$$
\begin{aligned}
& Y_{1}, \ldots, Y_{n}, \\
& \left\{A_{i j}, B_{k l} \mid i=1, \ldots, q: j=1, \ldots, p: k=1, \ldots, r: l=1, \ldots, q\right\}, \\
& W_{1}, \ldots, W_{s},
\end{aligned}
$$

which satisfies the following two conditions:

1. There exist $y_{1}, \ldots, y_{n}, w_{1}, \ldots, w_{s} \in R$ such that $y_{1}, \ldots, y_{n}$ forms a regular system of parameters and $\underline{y},\left\{a_{i j}, b_{k l} \mid i, j, k, l\right\}, \underline{w}$ is a solution of $\mathfrak{b}$.

2. If a regular local ring $E$ has a solution $\underline{y^{\prime}},\left\{a_{i j}^{\prime}, b_{k l}^{\prime} \mid i, j, k, l\right\}$, w of $\mathfrak{b}$ such that $y_{1}^{\prime}, \ldots, y_{n}^{\prime}$ forms a regular system of parameters of $E$, then the following sequence

$$
E^{p} \stackrel{\left(a_{i j}^{\prime}\right)}{\longrightarrow} E^{q} \stackrel{\left(b_{k l}^{\prime}\right)}{\longrightarrow} E^{r}
$$

is exact.

Proof. Let

$$
0 \longrightarrow L_{t} \longrightarrow L_{t-1} \longrightarrow \cdots \longrightarrow L_{0} \longrightarrow R^{p} \stackrel{\left(a_{i j}\right)}{\longrightarrow} R^{q} \stackrel{\left(b_{k l}\right)}{\longrightarrow} R^{r}
$$

be an exact sequence of $R$-free modules and $R$-linear maps. It is well known ([2]) that certain conditions about the rank and the grade of the determinantal ideals of the matrices corresponding to the above $R$-linear maps make the above sequence exact. Thus one can find $\mathfrak{b}$ immediately by Lemma 3.4 .

Q.E.D.

LEMma 3.6. Let $R$ be a regular local ring of dimension $n$ with the maximal ideal $\mathfrak{p}$ and $J=\left(g_{1}, \ldots, g_{l}\right)$ be a proper ideal of $R$. Assume that $\left\{g_{1}, \ldots, g_{l}\right\}$ minimally generates the ideal $J$. Then, for a sufficiently large $k$, there exists an ideal $\mathfrak{b}$ of the polynomial ring

$$
\mathbf{Z}\left[Y_{1}, \ldots, Y_{n}, G_{1}, \ldots, G_{l}, W_{1} \ldots W_{k}\right]
$$

which satisfies the following two conditions:

1. There exist $y_{1}, \ldots, y_{n}, w_{1}, \ldots, w_{k} \in R$ such that $y_{1}, \ldots, y_{n}$ forms a regular system of parameters of $R$ and $\underline{y}, \underline{g}, \underline{w}$ is a solution of $\mathfrak{b}$.

2. If a regular local ring $E$ has a solution $\underline{y^{\prime}}, \underline{g^{\prime}}, \underline{w^{\prime}}$ of $\mathfrak{b}$ such that $y_{1}^{\prime}, \ldots, y_{n}^{\prime}$ 
forms a regular system of parameters of $E$, then $\left\{g_{1}^{\prime}, \ldots, g_{l}^{\prime}\right\}$ minimally generates the ideal $\left(g_{1}^{\prime}, \ldots, g_{l}^{\prime}\right)$.

Proof. Let

$$
R^{p} \stackrel{\left(b_{i j}\right)}{\longrightarrow} R^{l} \stackrel{\left(g_{1} \cdots g_{l}\right)}{\longrightarrow} R
$$

be an exact sequence such that $b_{i j} \in \mathfrak{p}$ for any $i$ and $j$. Then the assertion is an immediate consequence of Lemma 3.5.

Q.E.D.

As we noted before, one can find an ideal $\mathfrak{b}$ inducing $h t_{E}\left(g_{1}^{\prime}, \ldots, g_{l}^{\prime}\right)=$ $\mathrm{ht}_{R}\left(g_{1}, \ldots, g_{l}\right)$ in the situation of Lemma 3.4 as follows:

Lemma 3.7. Let $R$ be a regular local ring of dimension $n$ and $\left(g_{1}, \ldots, g_{l}\right)$ be a proper ideal of $R$. Then, for a sufficiently large $k$, there exists an ideal $\mathfrak{b}$ of the polynomial ring

$$
\mathbf{Z}\left[Y_{1}, \ldots, Y_{n}, G_{1}, \ldots, G_{l}, W_{1}, \ldots, W_{k}\right]
$$

which satisfies the following two conditions:

1. There exist $y_{1}, \ldots, y_{n}, w_{1}, \ldots, w_{k} \in R$ such that $y_{1}, \ldots, y_{n}$ forms a regular system of parameters of $R$ and $\underline{y}, \underline{g}, \underline{w}$ is a solution of $\mathfrak{b}$.

2. If a regular local ring $E$ has a solution $\underline{y}^{\prime}, g^{\prime}, w^{\prime}$ of $\mathfrak{b}$ such that $y_{1}^{\prime}, \ldots, y_{n}^{\prime}$ forms a regular system of parameters of $E$, then $\mathrm{ht}_{E}\left(g_{1}^{\prime}, \ldots, g_{l}^{\prime}\right)=\mathrm{ht}_{R}\left(g_{1}, \ldots\right.$, $\left.g_{l}\right)$ is satisfied.

Proof. Put $h=\mathrm{ht}_{R}\left(g_{1}, \ldots, g_{l}\right)$. At first, assume $h=l$. Choosing $x_{1}, \ldots, x_{n-l}$ $\in R$ such that $\mathrm{ht}_{R}\left(g_{1}, \ldots, g_{l}, x_{1}, \ldots, x_{n-l}\right)=n$ and replacing $\left(g_{1}, \ldots, g_{l}\right)$ by $\left(g_{1}, \ldots, g_{l}, x_{1}, \ldots, x_{n-l}\right)$, we may assume $h=l=n$. Then assertion is obvious because $\sqrt{\left(g_{1}, \ldots, g_{l}\right)}=\left(y_{1}, \ldots, y_{n}\right)$.

Next assume $h<l$. Take $p_{1}, \ldots, p_{h} \in\left(g_{1}, \ldots, g_{l}\right)$ which satisfy $h=$ $\mathrm{ht}_{R}\left(p_{1}, \ldots, p_{h}\right)$. Then it is easy to see that there exists $c \in R$ such that the following two conditions are satisfied:

1. Either $\mu_{R}\left(\left(p_{1}, \ldots, p_{h}, c\right)\right)=h+1$ or $c=1$ is satisfied. $\left(\mu_{R}(*)\right.$ stands for the cardinary of a minimal generating set. It is well known that it does not depend on the choice of generators.)

2. $c \cdot\left(g_{1}, \ldots, g_{l}\right)^{\nu} \subseteq\left(p_{1}, \ldots, p_{h}\right)$ for $\nu \gg 0$.

Then the assertion is an immediate consequence of Lemma 3.4 and Lemma 3.6.

Q.E.D. 
Proof of Proposition 3.3. Owing to Lemma 3.7, it is sufficient to prove the following claim:

CLAIM 3.8. Let a be an ideal of the polynomial ring $\mathbf{Z}\left[Y_{1}, \ldots, Y_{n}, W_{1} \ldots\right.$ $W_{k}$ ] over $\mathbf{Z}$. Suppose that a regular local ring $B$ containing $\mathbf{Q}$ has a solution $\underline{y}, \underline{w}$ of a such that $y_{1}, \ldots, y_{n}$ forms a regular system of parameters of $B$. Then there exists a regular local ring $C$ which is essentially of finite type over a field of positive characteristic and has a solution $\underline{y}^{\prime}, \underline{w}^{\prime}$ of a such that $y_{1}^{\prime}, \ldots, y_{n}^{\prime}$ forms a regular system of $p a$ rameters of $C$.

We shall prove Claim 3.8 in two steps.

Step 1. Here we prove Claim 3.8 in the case where $B$ is essentially of finite type over a field of characteristic zero.

Then it is easy to see that we may put $B=K\left[t_{1}, \ldots, t_{n}\right]_{\left(t_{1}, \ldots, t_{n}\right)}$, where $K$ is an algebraically closed field of characteristic 0 and $t_{1}, \ldots, t_{n}$ are algebraically independent elements over $K$. Since $\left(t_{1}, \ldots, t_{n}\right) B=\left(y_{1}, \ldots, y_{n}\right) B$, we have $t_{i}=$ $\sum_{j=1}^{n} d_{i j} y_{j}(i=1, \ldots, n)$ with $d_{i j} \in B$ for $i, j=1, \ldots, n$. Let $\mathfrak{b}$ be the ideal of the polynomial ring

$$
\mathbf{Z}\left[\underline{Y}, \underline{W}, T_{1}, \ldots, T_{n},\left\{D_{i j} \mid i, j=1, \ldots, n\right\}\right]
$$

generated by $a$ and $\left\{T_{i}-\sum_{j=1}^{n} D_{i j} Y_{j} \mid i=1, \ldots, n\right\}$. Then $B$ has a solution $\underline{y}, \underline{w}$, $\underline{t},\left\{d_{i j} \mid i, j\right\}$ of $\mathfrak{b}$. Let $f$ be an element contained in $K\left[t_{1}, \ldots, t_{n}\right] \backslash\left(t_{1}, \ldots, t_{n}\right)$ such that $f y_{1}, \ldots, f y_{n}, f w_{1}, \ldots, f w_{n},\left\{f d_{i j} \mid i, j\right\}$ are contained in $K\left[t_{1}, \ldots, t_{n}\right]$. Put $y_{i}^{\prime \prime}=f y_{i}, w_{j}^{\prime \prime}=f w_{j}$ and $d_{i j}^{\prime \prime}=f d_{i j}$ for each $i, j$. Here note that $y_{1}^{\prime \prime}, \ldots, y_{n}^{\prime \prime} \in$ $\left(t_{1}, \ldots, t_{n}\right) \cdot K\left[t_{1}, \ldots, t_{n}\right]$. Let $D$ be a finitely generated $\mathbf{Z}$-algebra contained in $K$ such that $f, \underline{y^{\prime \prime}}, \underline{w^{\prime \prime}},\left\{d_{i j}^{\prime \prime} \mid i, j\right\} \in D\left[t_{1}, \ldots, t_{n}\right]$. Since $0 \neq f(0, \ldots, 0) \in D$, one can find a prime ideal $\mathfrak{q}$ of $D$ such that $\mathfrak{q} \not \supset f(0, \ldots, 0)$ and $\mathfrak{q} \cap \mathbf{Z} \neq(0)$. We denote by $\phi$ the composite map

$$
\begin{aligned}
D\left[t_{1}, \ldots, t_{n}, f^{-1}\right] \rightarrow(D / q)\left[t_{1}, \ldots, t_{n}, f^{-1}\right] & \rightarrow Q(D / q)\left[t_{1}, \ldots, t_{n}, f^{-1}\right] \\
& \rightarrow Q(D / q)\left[t_{1}, \ldots, t_{n}\right]_{\left(t_{1}, \cdots, t_{n}\right)},
\end{aligned}
$$

where $Q(D / \mathfrak{q})$ stands for the fractional field of $D / \mathfrak{q}$. Since $D\left[t_{1}, \ldots, t_{n}, f^{-1}\right]$ has a solution $y_{1}^{\prime \prime} / f, \ldots, y_{n}^{\prime \prime} / f, w_{1}^{\prime \prime} / f, \ldots, w_{k}^{\prime \prime} / f, t,\left\{d_{i j}^{\prime \prime} / f \mid i, j\right\}$ of $\mathfrak{b}, Q(D / \mathfrak{q})\left[t_{1}, \ldots\right.$, $\left.t_{n}\right]_{\left(t_{1}, \cdots, t_{n}\right)}$ also has a solution

$$
\frac{\phi\left(y_{1}^{\prime \prime}\right)}{\phi(f)}, \ldots, \frac{\phi\left(y_{n}^{\prime \prime}\right)}{\phi(f)}, \frac{\phi\left(w_{1}^{\prime \prime}\right)}{\phi(f)}, \ldots, \frac{\phi\left(w_{k}^{\prime \prime}\right)}{\phi(f)}, \underline{t},\left\{\frac{\phi\left(d_{i j}^{\prime \prime}\right)}{\phi(f)} \mid i, j\right\}
$$


of $\mathfrak{b}$. Here note that

$$
\frac{\phi\left(y_{1}^{\prime \prime}\right)}{\phi(f)}, \ldots, \frac{\phi\left(y_{n}^{\prime \prime}\right)}{\phi(f)} \in\left(t_{1}, \ldots, t_{n}\right) \cdot Q(D / \mathfrak{q})\left[t_{1}, \ldots, t_{n}\right]_{\left(t_{1}, \ldots t_{n}\right)} .
$$

Since $t_{1}, \ldots, t_{n}$ forms a regular system of parameters of $Q(D / \mathfrak{q})\left[t_{1}, \ldots, t_{n}\right]_{\left(t_{1}, \cdots, t_{n}\right)}$, so does $\phi\left(y_{1}^{\prime \prime}\right) / \phi(f), \ldots, \phi\left(y_{n}^{\prime \prime}\right) / \phi(f)$. Hence $Q(D / \mathfrak{q})\left[t_{1}, \ldots, t_{n}\right]_{\left(t_{1}, \cdots, t_{n}\right)}$ has a solution

$$
\frac{\phi\left(y_{1}^{\prime \prime}\right)}{\phi(f)}, \ldots, \frac{\phi\left(y_{n}^{\prime \prime}\right)}{\phi(f)}, \frac{\phi\left(w_{1}^{\prime \prime}\right)}{\phi(f)}, \ldots, \frac{\phi\left(w_{k}^{\prime \prime}\right)}{\phi(f)}
$$

of a satisfying the requirement.

Step 2. Here we shall prove Claim 3.8 in general. We may assume that $B$ is a complete regular local ring. As we have already seen in Step 1, we have only to show that there exists a regular local ring $R$ which is essentially of finite type over a field of characteristic 0 and has a solution $\underline{y^{\prime \prime}}, \underline{w^{\prime \prime}}$ of a such that $y_{1}^{\prime \prime}, \ldots, y_{n}^{\prime \prime}$ forms a regular system of parameters of $R$.

Since $B$ is a complete regular local ring with regular system of parameters $y_{1}, \ldots, y_{n}, B$ is isomorphic to the formal power series ring $K\left[\left[y_{1}, \ldots, y_{n}\right]\right]$, where $K$ is a field of characteristic 0 . Put $E=K\left[y_{1}, \ldots, y_{n}\right]_{\left(y_{1}, \cdots, y_{n}\right)} \subset B$. Then $B$ is the completion of $E$ with respect to the $\left(y_{1}, \ldots, y_{n}\right)$-adic topology. We denote by ${ }^{h} E$ the henselization (cf. [11]) of $E$. Then ${ }^{h} E$ has a solution $\underline{y}$, $\underline{w}^{\prime \prime}$ of a by Artin's approximation theorem ([1]). Then, by virtue of (43.9) in [11], one can find an intermediate ring $E \subseteq R \subseteq{ }^{h} E$ such that $w^{\prime \prime}$ are contained in $R, R$ is essentially of finite type over $E$, and ${ }^{h} E$ coincides with ${ }^{h} R$. Then it is easy to see that $R$ is a regular local ring essentially of finite type over $K$, and $R$ has a solution $\underline{y}, \underline{w^{\prime \prime}}$ of a such that $y_{1}, \ldots, y_{n}$ forms a regular system of parameters of $R$. Thus $R$ satisfies all requirements.

We have completed the proof of Proposition 3.3.

Q.E.D.

Remark 3.9. As we noted before, Proposition 3.3 allows us to make a reduction while preserving homological properties of a given local ring. For example, with notation as in Proposition 3.3 , if $B /\left(g_{1}, \ldots, g_{l}\right)$ is a Cohen-Macaulay ring whose non complete intersection locus has dimension at most 0 , then one can find $C$ and a solution $\underline{y^{\prime}}, \underline{x^{\prime}}, \underline{g^{\prime}}, \underline{w^{\prime}}$ of a (satisfying the requirements stated in Proposition 3.3) such that $C /\left(g_{1}^{\prime}, \ldots, g_{l}^{\prime}\right)$ is also a Cohen-Macaulay ring whose non complete intersection locus has dimension at most 0 as follows.

With notation as in Proposition 3.3 , assume that $B /\left(g_{1}, \ldots, g_{l}\right)$ is a 
Cohen-Macaulay ring. Let

$$
\text { P. : } 0 \rightarrow P_{n-d} \rightarrow \cdots \rightarrow P_{2} \stackrel{\phi}{\rightarrow} P_{1} \rightarrow P_{0}
$$

be the minimal $B$-free resolution of $B /\left(g_{1}, \ldots, g_{l}\right)$. (Here, note that, since it is Cohen-Macaulay, the length of the minimal $B$-free resolution is equal to $n-d$.) It is easy to see that the non complete intersection locus of $B /\left(g_{1}, \ldots, g_{l}\right)$ has dimension at most 0 if and only if

$$
\sqrt{I_{m-n+d}(\phi)+\left(g_{1}, \ldots, g_{l}\right)} \supseteq\left(y_{1}, \ldots, y_{n}\right),
$$

where $m=\operatorname{rank}_{B} P_{1}$ and $I_{m-n+d}(\phi)$ stands for the ideal generated by all the $m-n+d$ by $m-n+d$ minors of the matrix corresponding to the $B$-linear map $\phi$.

Furthermore assume that non complete intersection locus of $B /\left(g_{1}, \ldots\right.$, $g_{l}$ ) has dimension at most 0 . Then, replacing the pair $a \subseteq \mathbf{Z}[\underline{Y}, \underline{X}, \underline{G}, \underline{W}]$ by another one (cf. Lemma 3.5 ), we may suppose that $C /\left(g_{1}^{\prime}, \ldots, g_{n}^{\prime}\right)$ has the minimal $C$-free resolution

$$
\mathbf{P}^{\prime} .: 0 \rightarrow P_{n-d}^{\prime} \rightarrow \cdots \rightarrow P_{2}^{\prime} \stackrel{\phi^{\prime}}{\rightarrow} P_{1}^{\prime} \rightarrow P_{0}^{\prime}
$$

such that $\operatorname{rank}_{B} P_{i}=\operatorname{rank}_{C} P_{i}^{\prime}$ for each $i$ and

$$
\sqrt{I_{m-n+d}\left(\phi^{\prime}\right)+\left(g_{1}^{\prime}, \ldots, g_{l}^{\prime}\right)} \supseteq\left(y_{1}^{\prime}, \ldots, y_{n}^{\prime}\right) .
$$

Hence, one can find $C$ and a solution $\underline{y}^{\prime}, \underline{x^{\prime}}, \underline{g^{\prime}}, \underline{w^{\prime}}$ of a (satisfying the requirements stated in Proposition 3.3) such that $C /\left(g_{1}^{\prime}, \ldots, g_{l}^{\prime}\right)$ is a Cohen-Macaulay ring whose non complete intersection locus has dimension at most 0 .

Before proving (3) of Theorem 1.1, we need to prove two lemmas.

Lemma 3.10. Let $(R, \mathfrak{p})$ be a regular local ring of dimension $n$ and $\phi: R^{p} \rightarrow$ $R^{q}$ be the $R$-linear map of $R$-free modules corresponding to the $q$ by $p$ matrix $\left(b_{i j}\right)$. Assume $\ell_{R}(\operatorname{Coker} \phi)<\infty$. Then, for a sufficiently large $k$, there exists an ideal $\mathfrak{b}$ of the polynomial ring

$$
\mathbf{Z}\left[Y_{1}, \ldots, Y_{n},\left\{B_{i j} \mid i=1, \ldots, q: j=1, \ldots, p\right\}, W_{1}, \ldots, W_{k}\right]
$$

which satisfies the following two conditions:

1. There exist $y_{1}, \ldots, y_{n}, w_{1}, \ldots, w_{k} \in R$ such that $y_{1}, \ldots, y_{n}$ forms a regular system of parameters of $R$ and $\underline{y},\left\{b_{i j} \mid i, j\right\}, \underline{w}$ is a solution of $\mathfrak{b}$.

2. If a regular local ring $E$ has a solution $\underline{y}^{\prime},\left\{b_{i j}^{\prime} \mid i, j\right\}$, w $\underline{w}^{\prime}$ of $\mathfrak{b}$ such that $y_{1}^{\prime}, \ldots, y_{n}^{\prime}$ forms a regular system of parameters of $E$, then $\ell_{R}(\operatorname{Coker} \phi)=$ 
$\ell_{E}\left(\right.$ Coker $\left.\phi^{\prime}\right)$ holds, where $\phi^{\prime}: E^{p} \rightarrow E^{q}$ is the $E$-iinear map of $E$-free modules corresponding to the $q$ by $p$ matrix $\left(b_{i j}^{\prime}\right)$.

Proof. We shall prove this lemma by induction on $l=\ell_{R}($ Coker $\phi)$.

If $l=0$, then the sequence

$$
R^{p} \stackrel{\phi}{\longrightarrow} R^{q} \longrightarrow 0
$$

is exact. Hence the assertion is an immediate consequence of Lemma 3.5.

Next suppose $l>0$. Let $L$ be an $R$-submodule of Coker $\phi$ such that

$$
0 \longrightarrow L \longrightarrow \text { Coker } \phi \longrightarrow R / \mathfrak{p} \longrightarrow 0
$$

is exact. Let $\mathbf{K}$. be the Koszul complex with respect to $y_{1}, \ldots, y_{n} \in R$ and $\mathbf{L}$. be the minimal $R$-free resolution of $L$. Then there exist an acyclic $R$-free complex $\mathbf{H}$. and an exact sequence of $R$-free complexes

$$
0 \longrightarrow \text { L. } \longrightarrow \text { H. } \longrightarrow \text { K. } \longrightarrow 0
$$

such that the induced exact sequence

$$
0 \longrightarrow \mathrm{H}_{0} \text { (L.) } \longrightarrow \mathrm{H}_{0} \text { (H.) } \longrightarrow \mathrm{H}_{0}(\text { K.) } \longrightarrow 0
$$

coincides with the sequence (1). Let

$$
\text { Q. : } 0 \longrightarrow Q_{q} \longrightarrow \cdots \longrightarrow Q_{1} \longrightarrow Q_{0}
$$

be an $R$-free resolution of Coker $\phi$ such that the $R$-linear map $Q_{1} \rightarrow Q_{0}$ coincides with $\phi: R^{p} \rightarrow R^{q}$. Denoting by $\mathbf{P}$. the minimal $R$-free resolution of Coker $\phi$, we have exact sequences of $R$-free acyclic complexes

$$
\begin{aligned}
& 0 \longrightarrow \mathbf{P} . \longrightarrow \text { H. } \longrightarrow \text { A. } \longrightarrow 0, \\
& 0 \longrightarrow \text { P. } \longrightarrow \text { Q. } \longrightarrow \text { B. } \longrightarrow 0,
\end{aligned}
$$

where $\mathbf{A}$. and $\mathbf{B}$. are split exact sequences. Construct an ideal $\mathfrak{b} \in \mathbf{Z}\left[\underline{Y},\left\{B_{i j} \mid i\right.\right.$, $j\}, \underline{W}$ in order to preserve the acyclicity of $\mathbf{L}$., H., K., $\mathbf{P}$., the split exactness of A., B., and the exactness of (2), (3), (4). If a regular local ring $E$ has a solution $\underline{y}^{\prime}$, $\left\{b_{i j}^{\prime} \mid i, j\right\}, \underline{w^{\prime}}$ of $\mathfrak{b}$ such that $y_{1}^{\prime}, \ldots, y_{n}^{\prime}$ forms a regular system of parameters of $E$, we have an exact sequence

$$
0 \longrightarrow \mathrm{H}_{0}\left(\mathbf{L}^{\prime}\right) \longrightarrow \mathrm{H}_{0}\left(\mathbf{H}^{\prime}\right) \longrightarrow \mathrm{H}_{0}\left(\mathbf{K}^{\prime}\right) \longrightarrow 0,
$$

where $\mathbf{H}_{0}\left(\mathbf{H}^{\prime}.\right)=$ Coker $\phi^{\prime}$. By a suitable choice of $\mathfrak{b}$, we may assume that $\mathbf{K}^{\prime}$. remains the Koszul complex (with respect to $y_{1}^{\prime}, \ldots, y_{n}^{\prime} \in E$ ) and, therefore, 
$\ell_{E}\left(H_{0}\left(\mathbf{K}^{\prime}\right)\right)=1$. By the assumption of induction on $l$, enlarging the ideal $\mathfrak{b}$, we may suppose $\ell_{E}\left(\mathrm{H}_{0}\left(\mathbf{L}^{\prime}\right)\right)=\ell_{R}\left(\mathrm{H}_{0}(\mathbf{L}).\right)=l-1$. Hence we get $\ell_{A}\left(\mathrm{H}_{0}\left(\mathbf{H}^{\prime}\right)\right)=$ $\ell_{E}\left(\operatorname{Coker} \phi^{\prime}\right)=l$.

Q.E.D.

Lemma 3.11. Let $R$ be a regular local ring of dimension $n$ and put $B=$ $R /\left(g_{1}, \ldots, g_{l}\right)$, where $\left(g_{1}, \ldots, g_{l}\right)$ is a proper ideal of $R$. Let $a_{i j}$ 's and $b_{k l}$ 's be elements in $R$ such that

$$
B^{p} \stackrel{\overline{\left(a_{i j}\right)}}{\longrightarrow} B^{q} \stackrel{\overline{\left(b_{k l}\right)}}{\longrightarrow} B^{r}
$$

is a complex of $B$-free modules and $B$-linear maps. Assume that the homology group $H$ of the above complex has finite length. Then, for a sufficiently large $s$, there exists an ideal $\mathfrak{b}$ of the polynomial ring over $\mathbf{Z}$ with indeterminates

$$
\begin{aligned}
& Y_{1}, \ldots, Y_{n}, \\
& \mathrm{G}_{1}, \ldots, \mathrm{G}_{l}, \\
& \left\{A_{i j}, B_{k l} \mid i=1, \ldots, q: j=1, \ldots, p: k=1, \ldots, r: l=1, \ldots, q\right\}, \\
& W_{1}, \ldots, W_{s},
\end{aligned}
$$

which satisfies the following two conditions:

1. There exist $y_{1}, \ldots, y_{n}, w_{1}, \ldots, w_{s} \in R$ such that $y_{1}, \ldots, y_{n}$ forms a regular system of parameters of $R$ and $\underline{y}, \underline{g},\left\{a_{i j}, b_{k l} \mid i, j, k, l\right\}, \underline{w}$ is a solution of $\mathfrak{b}$.

2. If a regular local ring $E$ has a solution $\underline{y^{\prime}}, \underline{g^{\prime}},\left\{a_{i j}^{\prime}, b_{k l}^{\prime} \mid i, j, k, l\right\}, \underline{w^{\prime}}$ of $\mathfrak{b}$ such that $y_{1}^{\prime}, \ldots, y_{n}^{\prime}$ forms a regular system of parameters of $E$, then the following sequence

$$
\left(E /\left(g_{1}^{\prime}, \ldots, g_{l}^{\prime}\right)\right)^{p} \stackrel{\overline{\left(a_{i j}^{\prime}\right)}}{\longrightarrow}\left(E /\left(g_{1}^{\prime}, \ldots, g_{l}^{\prime}\right)\right)^{q} \stackrel{\overline{\left(b_{k l}^{\prime}\right)}}{\longrightarrow}\left(E /\left(g_{1}^{\prime}, \ldots, g_{l}^{\prime}\right)\right)^{r}
$$

is a complex whose homology group has length equal to $\boldsymbol{\ell}_{R}(H)$.

Proof. We denote by $\phi$ (resp. $\phi$ ) the $B$-linear map corresponding to the mat$\operatorname{rix}\left(\overline{a_{i j}}\right)\left(\right.$ resp. $\left.\left(\overline{b_{k l}}\right)\right)$. Put $C=\operatorname{Ker} \phi, D=\operatorname{Im} \phi, E=\operatorname{Coker} \phi, I=\operatorname{Ker} \phi, J=$ $\operatorname{Im} \phi$ and $K=$ Coker $\phi$. Let C., D., E., I., J. and $\mathbf{K}$. be the minimal $R$-free resolutions of $C, D, E, I, J$ and $K$ respectively. Then we have exact sequences of acyclic $R$-free complexes

$$
\begin{aligned}
& 0 \longrightarrow \text { C. } \longrightarrow \text { L. } \stackrel{\alpha}{\longrightarrow} \text { D. } \longrightarrow 0 \text {, } \\
& 0 \longrightarrow \text { D. } \stackrel{\beta}{\longrightarrow} \mathbf{L}^{2} . \longrightarrow \text { E. } \longrightarrow 0 \text {, } \\
& 0 \longrightarrow \text { I. } \stackrel{\zeta}{\longrightarrow} \mathbf{L}^{3} \stackrel{r}{\longrightarrow} \text { J. } \longrightarrow 0 \text {, }
\end{aligned}
$$




$$
0 \longrightarrow \mathbf{J} . \stackrel{\delta}{\longrightarrow} \mathbf{L}^{4} \longrightarrow \mathbf{K} . \longrightarrow 0
$$

where both $\mathbf{L}^{2}$. and $\mathbf{L}^{3}$. are finite $R$-free resolutions of $B^{q}, \mathbf{L}^{1}$. (resp. $\mathbf{L}^{4}$.) is that of $B^{p}$ (resp. $B^{r}$ ), and $\alpha, \beta, \zeta, \gamma, \delta$ are chain maps such that $\mathrm{H}_{0}(\beta \cdot \alpha)$ (resp. $\mathrm{H}_{0}(\delta \cdot \gamma)$ ) coincides with $\phi$ (resp. $\left.\phi\right)$. Let $\mathbf{O}$. be the minimal $R$-free resolution of $B$. Put

$$
\begin{aligned}
& \text { P. }=\mathbf{O}^{\oplus p}, \\
& \text { Q. }=\mathbf{O}^{\oplus q}, \\
& \text { R. }=\mathbf{O}^{\oplus r},
\end{aligned}
$$

Since $\mathbf{O}^{\oplus n}$ is the minimal $R$-free resolution of $B^{n}$ for each $n$, we have exact sequences of finite $R$-free acyclic complexes

$$
\begin{aligned}
& 0 \longrightarrow \mathbf{P} . \stackrel{\varepsilon}{\longrightarrow} \mathbf{L}^{1} \longrightarrow \mathbf{S} . \longrightarrow 0 \\
& 0 \longrightarrow \mathbf{T} . \longrightarrow \mathbf{L}^{2} \stackrel{\lambda}{\longrightarrow} \mathbf{Q} . \longrightarrow 0 \text {, } \\
& 0 \longrightarrow \mathbf{U} . \longrightarrow \mathbf{L}^{3} \stackrel{\pi}{\longrightarrow} \mathbf{Q} . \longrightarrow 0 \text {, } \\
& 0 \longrightarrow \mathbf{R} . \stackrel{\nu}{\longrightarrow} \mathbf{L}^{4} . \longrightarrow \text { V. } \longrightarrow 0 \text {, }
\end{aligned}
$$

where $\mathbf{S}$., T., $\mathbf{U}$. and $\mathbf{V}$. are finite $R$-free split exact sequences, and $\varepsilon, \lambda, \pi, \nu$ are chain maps such that $\mathrm{H}_{0}(\varepsilon), \mathrm{H}_{0}(\lambda), \mathrm{H}_{0}(\pi), \mathrm{H}_{0}(\nu)$ are the identities.

Since $\mathrm{H}_{0}(\lambda \cdot \beta \cdot \alpha \cdot \varepsilon): B^{p} \rightarrow B^{q}$ coincides with $\phi$, there exists an $R$-linear map $f: P_{0} \rightarrow Q_{1}$ which satisfies

$$
\lambda_{0} \cdot \beta_{0} \cdot \alpha_{0} \cdot \varepsilon_{0}-\left(a_{i j}\right)=q_{1} \cdot f,
$$

where $q_{1}: Q_{1} \rightarrow Q_{0}$ is the boundary map of $\mathbf{Q}$, and $\left(a_{i j}\right)$ is the $R$-linear map $R^{p}$ $\rightarrow R^{q}$ corresponding to the matrix $\left(a_{i}\right)$. (For a chain map $\eta$, we denote by $\eta_{0}$ the map in degree 0 of $\eta . P_{i}$ (resp. $Q_{i}$ ) stands for the module in degree $i$ of the complex $\mathbf{P}$. (resp. Q.).)

As in the same way as above, there exists an $R$-linear map $g: L_{0}^{3} \rightarrow L_{1}^{4}$ such that

$$
\nu_{0} \cdot\left(b_{k l}\right) \cdot \pi_{0}-\delta_{0} \cdot \gamma_{0}=l_{1} \cdot g,
$$

where $l_{1}: L_{1}^{4} \rightarrow L_{0}^{4}$ is the boundary map of $\mathbf{L}^{4}$.

Let

$$
\xi: \text { D. } \rightarrow \mathbf{I} \text {. }
$$

be a chain map such that $\mathrm{H}_{0}(\xi): D \rightarrow I$ coincides with the natural inclusion $D \hookrightarrow$ 
$I$. Then it is easy to see that the mapping cone of $\xi: \mathbf{D} . \rightarrow \mathbf{I}$. is a finite $R$-free resolution of the homology group $H$ and, in particular, the sequence

$$
D_{0} \oplus I_{1} \stackrel{\xi_{0}+i_{1}}{\longrightarrow} I_{0} \longrightarrow H \longrightarrow 0
$$

is exact, where $i_{1}: I_{1} \rightarrow I_{0}$ is the boundary map of $\mathbf{I}$..

Since $\mathrm{H}_{0}(\lambda \cdot \beta)=\mathrm{H}_{0}(\pi \cdot \zeta \cdot \xi)$, there exists an $R$-linear map $h: D_{0} \rightarrow Q_{1}$ such that

$$
\lambda_{0} \cdot \beta_{0}-\pi_{0} \cdot \zeta_{0} \cdot \xi_{0}=q_{1} \cdot h
$$

(Recall that $q_{1}: Q_{1} \rightarrow Q_{0}$ is the boundary map of $\mathbf{Q}$..)

Now construct an ideal $\mathfrak{b}$ to preserve the following properties:

- the split exactness of S., T., U., V.,

- the acyclicity of C., D., E., I., J., K., O., P., Q., R., L., $\mathbf{L}^{2}, \mathbf{L}_{.}^{3}, \mathbf{L}^{4}$,

- the equalities of (9), (10), (11), (16),(17), (18),

- the exactness of (5), (6), (7), (8), (12), (13), (14), (15),

- that $\xi$ is a chain map,

and to stabilize the length of the cokernel of $D_{0} \oplus I_{1} \stackrel{\xi_{0}+i_{1}}{\longrightarrow} I_{0}$. (cf. Lemma 3.5 and Lemma 3.10)

Suppose that a regular local ring $E$ has a solution $\underline{y}^{\prime}, g^{\prime},\left\{a_{i j}^{\prime}, b_{k l}^{\prime} \mid i, j, k, l\right\}$, $w^{\prime}$ of $\mathfrak{b}$ such that $y_{1}^{\prime}, \ldots, y_{n}^{\prime}$ forms a regular system of parameters of $E$. Then there exist split exact $E$-free sequences $\mathbf{S}^{\prime}$., $\mathbf{T}^{\prime}$, $\mathbf{U}^{\prime}, \mathbf{V}_{.}^{\prime}$, the acyclic finite $E$-free com-

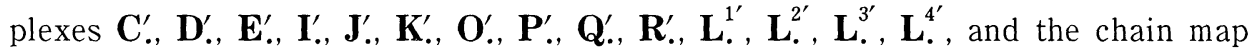
$\xi^{\prime}: \mathbf{D}^{\prime} \rightarrow \mathbf{I}^{\prime}$. such that

- the following sequences

$$
\begin{aligned}
& 0 \longrightarrow \mathbf{C}^{\prime} \longrightarrow \mathbf{L}^{1^{\prime}} \stackrel{\alpha^{\prime}}{\longrightarrow} \mathbf{D}^{\prime} . \longrightarrow 0, \\
& 0 \longrightarrow \mathbf{D}^{\prime} \stackrel{\beta^{\prime}}{\longrightarrow} \mathbf{L}^{2^{\prime}} \stackrel{\longrightarrow}{\longrightarrow} \mathbf{E}^{\prime} \longrightarrow 0 \text {, } \\
& 0 \longrightarrow \mathbf{I}^{\prime} \stackrel{\zeta^{\prime}}{\longrightarrow} \mathbf{L}^{3^{\prime}} \stackrel{r^{\prime}}{\longrightarrow} \mathbf{J}^{\prime} \stackrel{\longrightarrow}{\longrightarrow} \text {, } \\
& 0 \longrightarrow \mathbf{J}^{\prime} \stackrel{\delta^{\prime}}{\longrightarrow} \mathbf{L}^{4^{\prime}} \longrightarrow \mathbf{K}^{\prime} \longrightarrow 0 \text {, } \\
& 0 \longrightarrow \mathbf{P}^{\prime} \stackrel{\varepsilon^{\prime}}{\longrightarrow} \mathbf{L}^{1^{\prime}} \longrightarrow \mathbf{S}^{\prime} \longrightarrow 0 \\
& 0 \longrightarrow \mathbf{T}^{\prime} \longrightarrow \mathbf{L}^{2^{\prime}} \stackrel{\lambda^{\prime}}{\longrightarrow} \mathbf{Q}^{\prime} \longrightarrow 0 \text {, } \\
& 0 \longrightarrow \mathbf{U}^{\prime} \longrightarrow \mathbf{L}^{3^{\prime}} \stackrel{\pi^{\prime}}{\longrightarrow} \mathbf{Q}^{\prime} \longrightarrow 0 \text {, } \\
& 0 \longrightarrow \mathbf{R}^{\prime} \stackrel{\nu^{\prime}}{\longrightarrow} \mathbf{L}^{4^{\prime}} \longrightarrow \mathbf{V}^{\prime} \longrightarrow 0 \text {, }
\end{aligned}
$$


are exact,

- $\mathbf{O}^{\prime}$. is the minimal $E$-free resolution of $E /\left(g_{1}^{\prime}, \ldots, g_{l}^{\prime}\right)$,

$\cdot \mathbf{P}^{\prime}=\mathbf{O}_{.}^{\prime \oplus p}, \mathbf{Q}_{.}^{\prime}=\mathbf{O}_{.}^{\oplus q}, \mathbf{R}_{.}^{\prime}=\mathbf{O}_{.}^{\prime \oplus r}$,

- we have equations

$$
\begin{gathered}
\lambda_{0}^{\prime} \cdot \boldsymbol{\beta}_{0}^{\prime} \cdot \alpha_{0}^{\prime} \cdot \varepsilon_{0}^{\prime}-\left(a_{i j}^{\prime}\right)=q_{1}^{\prime} \cdot f^{\prime}, \\
\nu_{0}^{\prime} \cdot\left(b_{k l}^{\prime}\right) \cdot \pi_{0}^{\prime}-\delta_{0}^{\prime} \cdot \gamma_{0}^{\prime}=l_{1}^{\prime} \cdot g^{\prime}, \\
\lambda_{0}^{\prime} \cdot \boldsymbol{\beta}_{0}^{\prime}-\pi_{0}^{\prime} \cdot \zeta_{0}^{\prime} \cdot \xi_{0}^{\prime}=q_{1}^{\prime} \cdot h^{\prime},
\end{gathered}
$$

where $q_{1}^{\prime}: Q_{1}^{\prime} \rightarrow Q_{0}^{\prime}$ (resp. $l_{1}^{\prime}: L_{1}^{4^{\prime}} \rightarrow L_{0}^{4^{\prime}}$ ) is the boundary map of $\mathbf{Q}^{\prime}$. (resp. $\mathbf{L}^{4^{\prime}}$ ), and $f^{\prime}: P_{0}^{\prime} \rightarrow Q_{1}^{\prime}, g^{\prime}: L_{0}^{3^{\prime}} \rightarrow L_{1}^{4^{\prime}}, h^{\prime}: D_{0}^{\prime} \rightarrow Q_{1}^{\prime}$ are $R$-linear maps,

- the cokernel of $D_{0}^{\prime} \otimes I_{1}^{\prime} \stackrel{\xi_{0}^{\prime}+i_{1}^{\prime}}{\longrightarrow} I_{0}^{\prime}$ has length equal to $\ell_{R}(H)$, where $i_{1}^{\prime}: I_{1}^{\prime} \rightarrow I_{0}^{\prime}$ is the boundary map of $\mathbf{I}^{\prime}$.

Then we have the following exact sequences:

$$
\begin{aligned}
& 0 \longrightarrow \mathrm{H}_{0}\left(\mathbf{C}^{\prime}\right) \longrightarrow \mathrm{H}_{0}\left(\mathbf{L}_{.}^{1^{\prime}}\right) \stackrel{\mathrm{H}_{0}\left(\alpha^{\prime}\right)}{\longrightarrow} \mathrm{H}_{0}\left(\mathbf{D}^{\prime}\right) \longrightarrow 0 \\
& 0 \longrightarrow \mathrm{H}_{0}\left(\mathbf{D}^{\prime}\right) \stackrel{\mathrm{H}_{0}\left(\beta^{\prime}\right)}{\longrightarrow} \mathrm{H}_{0}\left(\mathbf{L}^{2^{\prime}}\right) \longrightarrow \mathrm{H}_{0}\left(\mathbf{E}^{\prime}\right) \longrightarrow 0 \\
& 0 \longrightarrow \mathrm{H}_{0}\left(\mathbf{I}^{\prime}\right) \stackrel{\mathrm{H}_{0}\left(\zeta^{\prime}\right)}{\longrightarrow} \mathrm{H}_{0}\left(\mathbf{L}^{3^{\prime}}\right) \stackrel{\mathrm{H}_{0}\left(r^{\prime}\right)}{\longrightarrow} \mathrm{H}_{0}\left(\mathbf{J}^{\prime}\right) \longrightarrow 0 \\
& 0 \longrightarrow \mathrm{H}_{0}\left(\mathbf{J}^{\prime}\right) \stackrel{\mathrm{H}_{0}\left(\delta^{\prime}\right)}{\longrightarrow} \mathrm{H}_{0}\left(\mathbf{L}^{4^{\prime}}\right) \longrightarrow \mathrm{H}_{0}\left(\mathbf{K}^{\prime}\right) \longrightarrow 0 \\
& 0 \longrightarrow \mathrm{H}_{0}\left(\mathbf{P}^{\prime} .\right) \stackrel{\mathrm{H}_{0}\left(\varepsilon^{\prime}\right)}{\longrightarrow} \mathrm{H}_{0}\left(\mathbf{L}^{1^{\prime}}\right) \longrightarrow 0 \\
& 0 \longrightarrow \mathrm{H}_{0}\left(\mathbf{L}^{2^{\prime}}\right) \stackrel{\mathrm{H}_{0}\left(\lambda^{\prime}\right)}{\longrightarrow} \mathrm{H}_{0}\left(\mathbf{Q}^{\prime}\right) \longrightarrow 0 \\
& 0 \longrightarrow \mathrm{H}_{0}\left(\mathbf{L}^{3^{\prime}}\right) \stackrel{\mathrm{H}_{0}\left(\pi^{\prime}\right)}{\longrightarrow} \mathrm{H}_{0}\left(\mathbf{Q}^{\prime}\right) \longrightarrow 0 \\
& 0 \longrightarrow \mathrm{H}_{0}\left(\mathbf{R}^{\prime}\right) \stackrel{\mathrm{H}_{0}\left(\nu^{\prime}\right)}{\longrightarrow} \mathrm{H}_{0}\left(\mathbf{L}^{4^{\prime}}\right) \longrightarrow 0
\end{aligned}
$$

By the equation (19), the composite map

$$
\begin{aligned}
\left(E /\left(g_{1}^{\prime}, \ldots, g_{l}^{\prime}\right)\right)^{p}=\mathrm{H}_{0}\left(\mathbf{P}^{\prime}\right) \stackrel{\mathrm{H}_{0}\left(\varepsilon^{\prime}\right)}{\longrightarrow} \mathrm{H}_{0}\left(\mathbf{L}_{.}^{1^{\prime}}\right) \stackrel{\mathrm{H}_{0}\left(\alpha^{\prime}\right)}{\longrightarrow} \mathrm{H}_{0}\left(\mathbf{D}^{\prime}\right) \stackrel{\mathrm{H}_{0}\left(\beta^{\prime}\right)}{\longrightarrow} \mathrm{H}_{0}\left(\mathbf{L}_{.}^{2^{\prime}}\right) \\
\stackrel{\mathrm{H}_{0}\left(\lambda^{\prime}\right)}{\longrightarrow} \mathrm{H}_{0}\left(\mathbf{Q}_{.}^{\prime}\right)=\left(E /\left(g_{1}^{\prime}, \ldots, g_{l}^{\prime}\right)\right)^{q}
\end{aligned}
$$

coincides with the $E /\left(g_{1}^{\prime}, \ldots, g_{l}^{\prime}\right)$-linear map corresponding to $\left(\overline{a_{i j}^{\prime}}\right)$. Hence the image of

$$
\left(E /\left(g_{1}^{\prime}, \ldots, g_{l}^{\prime}\right)\right)^{p} \stackrel{\overline{\left(a_{i j}^{\prime}\right)}}{\longrightarrow}\left(E /\left(g_{1}^{\prime}, \ldots, g_{l}^{\prime}\right)\right)^{q}
$$

coincides with $\operatorname{Im~} \mathrm{H}_{0}\left(\lambda^{\prime} \cdot \beta^{\prime}\right)$.

Furthermore by (20), the composite map 


$$
\begin{aligned}
\mathrm{H}_{0}\left(\mathbf{L}_{.}^{3^{\prime}}\right) \stackrel{\mathrm{H}_{0}\left(\pi^{\prime}\right)}{\longrightarrow} & \mathrm{H}_{0}\left(\mathbf{Q}^{\prime}\right) \\
& =\left(E /\left(g_{1}^{\prime}, \ldots, g_{l}^{\prime}\right)\right)^{q} \stackrel{\left(\frac{\left.b_{k l}^{\prime}\right)}{\longrightarrow}\right.}{\longrightarrow}\left(E /\left(g_{1}^{\prime}, \ldots, g_{l}^{\prime}\right)\right)^{r}=\mathrm{H}_{0}\left(\mathbf{R}_{.}^{\prime}\right) \stackrel{\mathrm{H}_{0}\left(\nu^{\prime}\right)}{\longrightarrow} \mathrm{H}_{0}\left(\mathbf{L}^{4^{\prime}}\right)
\end{aligned}
$$

coincides with $\mathrm{H}_{0}\left(\delta^{\prime} \cdot \gamma^{\prime}\right)$. Hence the kernel of

$$
\left(E /\left(g_{1}^{\prime}, \ldots, g_{l}^{\prime}\right)\right)^{q} \stackrel{\overline{\left(b_{k l}^{\prime}\right)}}{\longrightarrow}\left(E /\left(g_{1}^{\prime}, \ldots, g_{l}^{\prime}\right)\right)^{r}
$$

coincides with $\operatorname{Im~} \mathrm{H}_{0}\left(\pi^{\prime} \cdot \zeta^{\prime}\right)$.

On the other hand, by $(21)$, we have $\mathrm{H}_{0}\left(\lambda^{\prime} \cdot \beta^{\prime}\right)=\mathrm{H}_{0}\left(\pi^{\prime} \cdot \zeta^{\prime} \cdot \xi^{\prime}\right)$. Therefore the following sequence

$$
\left(E /\left(g_{1}^{\prime}, \ldots, g_{l}^{\prime}\right)\right)^{p} \stackrel{\overline{\left(a_{i j}^{\prime}\right)}}{\longrightarrow}\left(E /\left(g_{1}^{\prime}, \ldots, g_{l}^{\prime}\right)\right)^{q} \stackrel{\overline{\left(b_{k l}^{\prime}\right)}}{\longrightarrow}\left(E /\left(g_{1}^{\prime}, \ldots, g_{l}^{\prime}\right)\right)^{r}
$$

is a complex whose homology group is isomorphic to the cokernel of $\mathrm{H}_{0}\left(\xi^{\prime}\right)$. Since the cokernel of $\mathrm{H}_{0}\left(\xi^{\prime}\right)$ coincides with that of $D_{0}^{\prime} \oplus I_{1}^{\prime} \stackrel{\xi_{0}^{\prime}+i_{0}^{\prime}}{\longrightarrow} I_{0}^{\prime}$, the homology group of the complex (22) has length equal to $\ell_{R}(H)$.

Q.E.D.

Now we start to prove (3) of Theorem 1.1 .

Proof of (3) of Theorem 1.1 (in the case where $A$ contains $\mathbf{Q}$ ). Assume the contrary, i.e., there exists a Cohen-Macaulay local ring $A$ (cf. Remark 2.2), containing $\mathbf{Q}$, whose non complete intersection locus has dimension at most 0 such that $A$ has finitely generated modules $M$ and $N$ which satisfy the assumption of (3) of Theorem 1.1, and $\chi(M, N) \leq 0$.

Then there exists a Cohen-Macaulay local ring $A^{\prime}$, essentially of finite type over a field of positive characteristic, whose non complete intersection locus has dimension at most 0 such that $A^{\prime}$ has finitely generated modules $M^{\prime}$ and $N^{\prime}$ which satisfy the assumption of (3) of Theorem 1.1, and

$$
\ell_{A}\left(\operatorname{Tor}_{i}^{A}(M, N)\right)=\ell_{A^{\prime}}\left(\operatorname{Tor}_{i}^{A^{\prime}}\left(M^{\prime}, N^{\prime}\right)\right)
$$

for each $i$ (cf. Proposition 3.3, Lemma 3.7, Remark 3.9 and Lemma 3.11). Here note that $A^{\prime}$ is equi-dimensional and a homomorphic image of a regular local ring.

But, it is a contradiction because we have already proved (3) of Theorem 1.1 in the case where $A$ contains a field of positive characteristic in Section 2. Q.E.D.

\section{REFERENCES}

[1] M. Artin, Algebraic approximation of structures over complete local rings, Publ. Math. I.H.E.S., 36 (1969) 23-58. 
[2] D. A. Buchsbaum and D. Eisenbud, What makes a complex exact?, J. Algebra, 25 (1973), 259-268.

[ 3 ] S. P. Dutta, Frobenius and multiplicities, J. Algebra, 85 (1983), 424-448.

[4] - A special case of positivity, Proc. Amer. Math. Soc., 103 (1988), 344-346.

[5] - M. Hochster and J. E. MacLaughlin, Modules of finite projective dimension with negative intersection multiplicities, Invent. Math., 79 (1985), 253-291.

[6] W. Fulton, Intersection Theory, Springer-Verlag, Berlin, New York, 1984.

[7] H. Gillet and C. Soulé, K-théorie et nullité des multiplicites d'intersection, C. R. Acad. Sci. Paris Ser. I Math., 300 (1985), 71-74.

[ 8 ] F. Hirzebruch, Topological methods in algebraic geometry, 1956, Grundlehren der math. Wissenschaften, Vol. 131, Third enlarged edition, Springer-Verlag, 1966.

[9] M. Hochster, Topics in the homological theory of modules over local rings, C. B. M. S. Regional Conference Series in Math., 24. Amer. Math. Soc. Providence, R. I., 1975.

[10] K. Kurano, An approach to the characteristic free Dutta multiplicities, J. Math. Soc. Japan, 45 (1993), 369-390.

[11] M. Nagata, Local Rings, Interscience Tracts in Pure and Appl. Math., Wiley, New York, 1962.

[12] P. Roberts, The vanishing of intersection multiplicities and perfect complexes, Bull. Amer. Math. Soc., 13 (1985), 127-130.

[13] - MacRae invariant and the first local chern character, Trans. Amer. Math. Soc., 300 (1987), 583-591.

[14] - Local Chern characters and intersection multiplicities, Proc. of Symposia in Pure Math., 46 (1987), 389-400.

[15] - Intersection theorems, Commutative algebra, Proc. Microprogram, June 15-July 12, 1987, Math. Sci. Res. Inst. Publ., no. 15, Springer-Verlag, Berlin, Heidelberg, London, Paris, Tokyo, 1989, 417-436.

[16] - Local Chern classes, multiplicities, and perfect complexes, Société Mathématique de France Mémoire, no. 38 (1989), 145-161.

[17] J-P. Serre, Algèbre locale. Multiplicités, Lect. Note in Math., vol. 11, SpringerVerlag, Berlin, New York, 1965.

Department of Mathematics

Tokyo Metropolitan University

Minami-Ohsawa 1-1

Hachioji, Tokyo, 192-03

Japan

e-mail address: kurano@math.metro-u. ac.jp 